Pure and Applied Mathematics Quarterly

Volume 1, Number 1, 1-13, 2005

\title{
On the values of Artin $L$-functions
}

\author{
Benedict H. Gross
}

\section{Information}

I wrote this paper in 1979, as an attempt to extend the results of Borel [2] on zeta functions at negative integers to Artin $L$-functions. The conceptual framework was provided by Tate's formulation [10] of Stark's conjectures. What I needed was a workable definition of the regulator homomorphism in complex $K$-theory. I discussed this with Borel at the Institute, first over lunch and then in his office. It is an honor to dedicate this paper to his memory.

Using results of Bloch and Thurston, I was able to treat the special case of Dirichlet $L$-series at $s=-1$. I had the hope of treating Dirichlet $L$-series at all negative integers, where the order of vanishing is either zero or one, but was unable to construct the required "cyclotomic classes" in $K$-theory. This was done by Beilinson [11], who also found the generalization of my conjecture, and the conjectures of Deligne [6] on special values, to all motivic $L$-functionss.

I didn't publish this paper, but it has circulated as a preprint for 25 years. For reasons of historical interest, I decided to publish it in its original form here. I have updated the references, and added some comments on the recent literature at the end of the paper.

\section{Higher logarithms}

If $A$ is a commutative ring with unit, we let $K_{i} A, i>0$, be the abelian groups defined by Quillen [9]. Then

$$
K_{i} A=\pi_{i}\left(B G L(A)^{+}\right)
$$

Received September 24, 2004. 
and one has a canonical isomorphism:

$$
K_{i} A \otimes \mathbb{Q} \simeq \operatorname{Prim} H_{i}(G L(A), \mathbb{Q}) .
$$

In this section we will define canonical homomorphisms

$$
e_{2 m+1}: K_{2 m+1} \mathbb{C} \rightarrow \mathbb{R} .
$$

If we identify $K_{1} \mathbb{C} \simeq \mathbb{C}^{*}$ then $e_{1}$ becomes the homomorphism

$$
\begin{aligned}
\mathbb{C}^{*} & \rightarrow \mathbb{R} \\
z \mapsto \log |z| & =\operatorname{Re}(\log z) .
\end{aligned}
$$

Similarly, $e_{3}$ may be identified with the imaginary part of the dilogarithm. In general, if $\alpha \mapsto \bar{\alpha}$ is the involution on $K_{2 m+1} \mathbb{C}$ induced by complex conjugation, we have

$$
e_{2 m+1}(\bar{\alpha})=(-1)^{m} e_{2 m+1}(\alpha) .
$$

To define $e_{2 m+1}$ we will use Quillen's isomorphism (1.2) and construct an (indecomposible) class

$$
c_{2 m+1} \in H^{2 m+1}(G L(\mathbb{C}), \mathbb{R}) .
$$

This class will be in the image of the map

$$
H_{c}^{*}(G L(\mathbb{C}), \mathbb{R}) \underset{f}{\longrightarrow} H^{*}(G L(\mathbb{C}), \mathbb{R}),
$$

where $H_{c}^{*}$ denotes the continuous group cohomology of of $G L(\mathbb{C})$.

For $n>m$ let $X_{n}$ be the symmetric space

$$
X_{n}=U_{n} \backslash U_{n} \times U_{n},
$$

where $U_{n}$ is the unitary group of degree $n$, embedded in the product along the diagonal. Since $X_{n}$ is the "compact dual" of $G L_{n}(\mathbb{C})$, one has a canonical isomorphism [3]

$$
H_{\text {top }}^{*}\left(X_{n}, \mathbb{R}\right) \underset{g}{\stackrel{\sim}{\longrightarrow}} H_{c}^{*}\left(G L_{n}(\mathbb{C}), \mathbb{R}\right) .
$$

Complex conjugation acts as an involution on both exterior algebras; if $u$ is a topological class of pure degree

$$
\overline{g(u)}=(-1)^{\operatorname{deg} u} \cdot g(\bar{u}) .
$$


The integral cohomology of $X_{n}$ is a free exterior algebra, generated by the classes of odd spheres [4]:

$$
H_{\text {top }}^{*}\left(X_{n}, \mathbb{Z}\right) \simeq \Lambda_{\mathbb{Z}}^{*}\left(u_{1}, u_{3}, \ldots, u_{2 n-1}\right)
$$

We have

$$
\bar{u}_{2 m-1}=(-1)^{m} \cdot u_{2 m-1}
$$

as complex conjugation on $\mathbb{C}^{m}$ induces an automorphism of $S^{2 m-1}$ of degree $(-1)^{m}$. Setting

$$
v_{2 m+1}=g\left(u_{2 m+1}\right)
$$

and passing to the limit over $n[3]$ gives an isomorphism

$$
H_{c}^{*}(G L(\mathbb{C}), \mathbb{R}) \simeq \Lambda_{\mathbb{R}}^{*}\left(v_{1}, v_{3}, v_{5}, \ldots\right) .
$$

Furthermore, by combining (1.9) and (1.11) we obtain

$$
\bar{v}_{2 m+1}=(-1)^{m} \cdot v_{2 m+1} .
$$

Finally, let

$$
c_{2 m+1}=2 \pi \cdot f\left(v_{2 m+1}\right)
$$

where $f$ is the map in (1.6). This is the canonical class which corresponds to the honomorphisms $e_{2 m+1}$. An explicit cocycle for $c_{2 m+1}$ can be constructed geometrically, using the methods of Dupont [7].

\section{The theorems of Dirichlet and Borel}

Let $F$ be a number field of finite degree $n$ over $\mathbb{Q}$. Let $A$ be the ring of integers in $F$. Let

$$
Y=\operatorname{Hom}\left(R_{F / Q} \mathbb{G}_{m}, \mathbb{G}_{m / \mathbb{C}}\right) \simeq \mathbb{Z}^{\operatorname{Hom}(F, \mathbb{C})} .
$$

We have an exact sequence

$$
\begin{gathered}
0 \rightarrow Y_{0} \rightarrow Y \stackrel{\text { deg }}{\longrightarrow} \mathbb{Z} \rightarrow 0 \\
\sum a_{\phi} \cdot \phi \mapsto a_{\phi} .
\end{gathered}
$$

The groups $\operatorname{Aut}(F)$ and $\operatorname{Aut}_{\mathbb{R}} \mathbb{C} \simeq\langle c\rangle$ act on $Y$ and stabilize $Y_{0}$; they both act trivially on the quotient $Y / Y_{0} \simeq \mathbb{Z}$. 
Let $m$ be a nonnegative integer. Any complex embedding $\phi: F \rightarrow \mathbb{C}$ induces a map $\phi_{*}: K_{2 m+1} A \rightarrow K_{2 m+1} \mathbb{C}$ by functorality. Define the homomorphism

$$
\begin{aligned}
K_{2 m+1} & \underset{\lambda_{2 m+1}}{\longrightarrow} Y \otimes \mathbb{R} \\
\alpha & \mapsto \sum_{\phi} e_{2 m+1}\left(\phi_{*}(\alpha)\right) \cdot \phi,
\end{aligned}
$$

where $e_{2 m+1}$ is the homomorphism described in Section 1. By (1.4) the image of $\lambda_{2 m+1}$ lies in the subspace $(Y \otimes \mathbb{R})^{(-1)^{m}}$, where \pm denotes the eigenspaces of $c$. If $m=0$ then $K_{1} A \simeq A^{*}$ and $e_{1}\left(\phi_{*}(\alpha)\right)=\log \left|\phi_{*}(\alpha)\right|$. Consequently the image of $\lambda_{1}$ lies in the subspace $\left(Y_{0} \otimes \mathbb{R}\right)^{+}$, by the product formula.

Theorem 2.4 (Dirichlet, cf. [5]). The map $\lambda_{1}$ induces an isomorphism

$$
K_{1} A \otimes \mathbb{R} \underset{\lambda_{1}}{\stackrel{\sim}{\longrightarrow}}\left(Y_{0} \otimes \mathbb{R}\right)^{+} .
$$

Theorem 2.5 (Borel, [2], [3]). For $m>0$ the map $\lambda_{2 m+1}$ induces an isomor-

phism

$$
K_{2 m+1} A \otimes \mathbb{R} \underset{\lambda_{2 m+1}}{\stackrel{\sim}{\longrightarrow}}(Y \otimes \mathbb{R})^{(-1)^{m}} .
$$

The above isomorphisms are equivariant for $\operatorname{Aut}(F)$.

\section{Artin $L$-series}

Let $k$ be a number field, and let $F$ be a finite Galois extension of $k$. Let $G=$ $\operatorname{Gal}(F / k)$; if $w$ is a place of $k$ let $G_{w}$ be a decomposition group for $w$ in $G$.

Let $E$ be a finite extension field of $\mathbb{Q}$. Let $V$ be a vector space of finite dimension over $E$ which admits a linear action of $G$ :

$$
\rho: G \rightarrow \operatorname{Aut}_{E}(V)
$$

The class of this representation corresponds to an Artin motive over $k$ with coefficients in $E$ [6].

For any $s \in \operatorname{Hom}(E, \mathbb{C})$ we obtain a complex representation

$$
\rho^{\sigma}: G \rightarrow \operatorname{Aut}_{\mathbb{C}}\left(V^{\sigma}\right)
$$


by change of base. Let $L\left(V^{\sigma}, s\right)$ be its Artin $L$-series, and let

$$
L(V, s)=\left(\ldots, L\left(V^{\sigma}, s\right), \ldots\right)_{\operatorname{Hom}(E, \mathbb{C})} .
$$

This is a meromorphic function on $\mathbb{C}$ with values in $\mathbb{C} \otimes E \simeq \mathbb{C}^{\operatorname{Hom}(E, \mathbb{C})}$.

For $m \leq 0$ the order of $L\left(V^{\sigma}, s\right)$ at $s=-m$ is independent of $\sigma$. Let $d_{m}(V)$ be this order; we have the formulas

$$
\begin{gathered}
d_{0}(V)=\sum_{w \mid \infty} \operatorname{dim} V^{G_{w}}-\operatorname{dim} V^{G} . \\
d_{m}(V)=\sum_{\substack{\text { complex } \\
w \mid \infty}} \operatorname{dim} V^{G_{w}}+\sum_{\substack{\text { real } \\
w \mid \infty}} \operatorname{dim} V^{G_{w}} \quad \begin{array}{l}
m \text { even } \\
m>0
\end{array} \\
d_{m}(V)=\sum_{\substack{\text { complex } \\
w \mid \infty}} \operatorname{dim} V^{G_{w}}+\sum_{\substack{\text { real } \\
w \mid \infty}} \operatorname{dim}\left(V / V^{G_{w}}\right) \quad m \text { odd. }
\end{gathered}
$$

We define

$$
L(V,-m)^{*}=\lim _{s \rightarrow-m} \frac{L(V, s)}{(s+m)^{d_{m}(V)}}
$$

in $(\mathbb{C} \otimes E)^{*}$, and wish to conjecture its value modulo $E^{*}$.

The theorems of Dirichlet and Borel, applied to the field $F$, give us canonical $G$-isomorphisms:

$$
\begin{gathered}
K_{1} A \otimes \mathbb{R} \underset{\lambda_{1}}{\stackrel{\sim}{\longrightarrow}}\left(Y_{0} \otimes \mathbb{R}\right)^{+} \\
K_{2 m+1} A \otimes \mathbb{R} \underset{\lambda_{2 m+1}}{\stackrel{\sim}{\longrightarrow}}(Y \otimes \mathbb{R})^{(-1)^{m}} \quad m>0 .
\end{gathered}
$$

We may therefore choose $G$-isomorphisms of the underlying $\mathbb{Q}$-vector spaces:

$$
\begin{aligned}
& K_{1} A \otimes \mathbb{Q} \underset{\phi_{1}}{\stackrel{\sim}{\sim}}\left(Y_{0} \otimes \mathbb{Q}\right)^{+} \\
& K_{2 m+1} A \otimes \mathbb{Q} \underset{\phi_{2 m+1}}{\sim}(Y \otimes \mathbb{Q})^{(-1)^{m}} \quad m>0 .
\end{aligned}
$$

Let

$$
W_{2 m+1}= \begin{cases}\left(Y_{0} \otimes \mathbb{C}\right)^{+} & \text {if } m=0 \\ (Y \otimes \mathbb{C})^{(-1)^{m}} & \text { if } m>0\end{cases}
$$


Then $W_{2 m+1} \otimes V$ is a free $(\mathbb{C} \otimes E)$-module. The group $G$ acts linearly and one has

$$
\operatorname{rank}_{\mathbb{C} \otimes E}\left(W_{2 m+1} \otimes V\right)^{G}=d_{m}(V)
$$

by Frobenius reciprocity.

The autmorphism $\left(\lambda_{2 m+1} \circ \phi_{2 m+1}\right) \otimes 1$ is $\mathbb{C} \otimes E$-linear and commutes with the action of $G$. Define

$$
c(V, 2 m+1)=\operatorname{det}\left(\left(\lambda_{2 m+1} \circ \phi_{2 m+1}\right) \otimes 1 \mid\left(W_{2 m+1} \otimes V\right)^{G}\right) .
$$

Modulo $E^{*}$ this is independent of the choice of $\phi_{2 m+1}$.

Conjecture 3.11. For $m \geq 0$ the value $L(V,-m)^{*}$ is equal to the product of $c(V, 2 m+1)$ by an element of $E^{*}$.

\section{Remarks}

For $m=0$, Conjecture 3.11 is Stark's conjecture, as formulated by Tate [10]. It is known to be true in the following cases:

$$
\begin{array}{ll}
V \text { has a rational character } & \text { (Dirichlet, Ono, Lichtenbaum, Tate) } \\
V \text { is abelian and } k=\mathbb{Q} & \text { (Dirichlet, Lerch). } \\
V \text { is abelian and } k=\mathbb{Q}(\sqrt{-D}) & \text { (Kronecker). } \\
d_{0}(V)=0 & \text { (Siegel). }
\end{array}
$$

When $d_{m}(V)=0$, Conjecture 3.11 is a special case of Deligne's conjecture on the critical values of motivic $L$-series [6]. This case is known to be true by results of Siegel.

When $m>0$ and $d_{m}(V)>0$ less is known. For the trivial representation one has the much sharper conjectures of Lichetenbaum [8], and conjecture 3.11 was proved by Borel [2]. One deduces that (3.11) is true whenever $V$ has the form $\oplus m_{i} \operatorname{Ind}_{H_{i}}^{G} l_{H_{i}}$ with $m_{i} \in \mathbb{Z}$.

It would be very interesting to prove (3.11) either for all $V$ with rational character, or for all abelian representations $V$ of $\operatorname{Gal}(\overline{\mathbb{Q}} / \mathbb{Q})$. 
We will show how the methods of Bloch [1] can be used to settle the latter case in the affirmative when $m=1$.

\section{Character sums}

For $k>0$ and $|z| \leq 1$ define

$$
D_{k}(z)=\sum_{n \geq 1} \frac{z^{n}}{n^{k+1}} .
$$

Theorem 5.1. Let $k \geq 1$ be an integer. If $\chi$ is any Dirichlet character (mod f) then

$$
\sum_{(\mathbb{Z} / f)^{*}} \chi(a) D_{k}\left(e^{2 \pi i a / f}\right) \neq 0
$$

Corollary 5.2. Let $\operatorname{dil} \log (z)=\operatorname{Im} D_{1}(z)$. If $\chi$ is any odd Dirichlet character $(\bmod f)$ then

$$
\sum_{(\mathbb{Z} / f)^{*}} \chi(a) \operatorname{dilog}\left(e^{2 \pi i a / f}\right) \neq 0
$$

Proof of Corollary. Since $D_{k}(\bar{z})=\overline{D_{k}(z)}$, the real part of $D_{1}(z)$ is an even function. Since $\chi$ is assumed odd:

$$
\sum \chi(a) D_{1}\left(e^{2 \pi i a / f}\right)=i \cdot \sum \chi(a) \operatorname{dilog}\left(e^{2 \pi i a / f}\right) .
$$

Thus the Corollary follows from the theorem.

Translation. $\operatorname{dilog}\left(e^{2 \pi i a / f}\right)=2 \mathfrak{h}(\pi a / f)$ when $\mathfrak{h}$ is Lobachevsky's function.

Proof of Theorem. First assume $\chi$ is a primitive character $(\bmod f)$, and let $L(s, \chi)=\sum_{n \geq 1} \chi(n) n^{-s}$ be its Dirichlet $L$-series $(s>1)$.

Define the Fourier transform of $\chi$ on $\mathbb{Z} / f$ by

$$
\hat{\chi}(a)=\frac{1}{f} \sum_{(\mathbb{Z} / f)^{*}} \chi(b) e^{2 \pi i a b / f} .
$$


Since $\chi$ is primitive, $\hat{\chi}(a)=0$ unless $(a, f)=1$. Hence

$$
\chi(n)=\sum_{(\mathbb{Z} / f)^{*}} \hat{\chi}(a) e^{-2 \pi i a n / f} .
$$

by Fourier inversion. Putting this formula into the $L$-series, we obtain

$$
L(s, \chi)=\sum_{(\mathbb{Z} / f)^{*}} \hat{\chi}(a) D_{s-1}\left(e^{-2 \pi i a / f}\right) .
$$

Define the Gauss sum

$$
g(\chi)=\frac{1}{f} \sum_{(\mathbb{Z} / f)^{*}} \chi(a) e^{2 \pi i a / f} .
$$

Then making the change of variables $a \mapsto a b$, we see that $g(\chi)=\chi(a) \hat{\chi}(a)$ for all $(a, f)=1$. Consequently if $k \geq 1$ :

$$
L(1+k, \chi)=g(\chi) \sum_{(\mathbb{Z} / f)^{*}} \chi^{-1}(a) D_{k}\left(e^{-2 \pi i a / f}\right) .
$$

But $L(s, \chi)$ admits an Euler product which converges for $s>1$ :

$$
L(s, \chi)=\prod_{p}\left(1-\chi(p) p^{-s}\right)^{-1} .
$$

Consequently, $L(1+k, \chi) \neq 0$ and by $(5.1)$ :

$$
\sum_{(\mathbb{Z} / f)^{*}} \chi^{-1}(a) D_{k}\left(e^{-2 \pi i a / f}\right) \neq 0 .
$$

Now start with the primitive character $\chi^{-1}$ to obtain

$$
\sum_{(\mathbb{Z} / f)^{*}} \chi(a) D_{k}\left(e^{2 \pi i a / f}\right)=\chi(-1) \sum_{(\mathbb{Z} / f)^{*}} \chi(a) D_{k}\left(e^{-2 \pi i a / f}\right) \neq 0 .
$$

This completes the proof when $\chi$ is primitive $(\bmod f)$.

Now assume $g \mid f$ and $\chi$ comes from a primitive character $(\bmod g)$. Since the function $D_{k}: S^{1} \rightarrow \mathbb{C}$ is a distribution of weight $k$, we have the formula: 


$$
(f / g)^{k} \sum_{(\mathbb{Z} / f)^{*}} \chi(a) D_{k}\left(e^{2 \pi i a / f}\right)=\prod_{\substack{p \mid f \\ p \nmid g}}\left(1-\chi(p) p^{k}\right) \cdot \sum_{(\mathbb{Z} / g)^{*}} \chi(b) D_{k}\left(e^{2 \pi i b / g}\right) .
$$

The sum on the right-hand side is nonzero, by our previous argument. So is the product, as $\chi(p)$ is a root of unity and $k \geq 1$. This completes the proof.

\section{Notes:}

1) The result of Theorem 5.1 also holds when $k=0$, if we assume that $\chi$ is primitive and $\chi \neq 1$.

2 ) The function $L(s, \chi)$ satisfies a functional equation, which relates the value at $s=k+1$ to the leading term in the Taylor expansion at $s=-k$. For example, if $\chi$ is primitive and $\chi(-1)=(-1)^{k}$ then

$$
\begin{gathered}
L(-k, \chi)=0 \\
L^{\prime}(-k, \chi)=-\frac{1}{2} \frac{f^{k} k !}{(2 \pi i)^{k}} \sum_{(\mathbb{Z} / f)^{*}} \chi(a) D_{k}\left(e^{2 \pi i a / f}\right) .
\end{gathered}
$$

\section{$6 \quad K_{3}$ of abelian fields}

Let $F=\mathbb{Q}\left(\mu_{f}\right)$ and let $A=\mathbb{Z}\left[\mu_{f}\right]$. The Artin map gives a canonical isomorphism:

$$
\begin{aligned}
(\mathbb{Z} / f)^{*} & \simeq \operatorname{Gal}(F / \mathbb{Q}) \\
b & \rightarrow \operatorname{Frob}(b) \quad \text { where } \quad \zeta^{\operatorname{Frob}(b)}=\zeta^{b} .
\end{aligned}
$$

Fix an embedding $\phi: F \rightarrow \mathbb{C}$; this induces a map $\phi_{*}: K_{3} A \rightarrow K_{3} \mathbb{C}$ by functorality. Recall the map $e_{3}: K_{3} \mathbb{C} \rightarrow \mathbb{R}$ defined in $\S 1$.

Theorem 6.1 (Bloch, Thurston). For $a \in(\mathbb{Z} / f)^{*}$ there exist elements $C_{f}(a)$ in $K_{3} A \otimes \mathbb{Q}$ which satisfy

1) $C_{f}(a)^{\operatorname{Frob}(b)}=C_{f}(a b)$, 
2) $C_{f}(-a)=-C_{f}(a)$,

3) $e_{3}\left(\phi_{*} C_{f}(a)\right)=\frac{1}{2} \operatorname{dil} \log \left(e^{2 \pi i a / f}\right)$.

These properties (and the choice of $\phi$ ) completely determine the elements $C_{f}(a)$.

We will combine this result with Corollary 5.2 to prove the following result.

Theorem 6.2. 1) For any character $\chi(\bmod f)$ the element $C_{\chi}=\sum_{(\mathbb{Z} / f)^{*}} \chi(a) \otimes$ $C_{f}(a)$ spans the $\chi^{-1}$-eigenspace of $\mathbb{C} \otimes K_{3} A$.

2) The elements $\left\{C_{f}(a): a \in(\mathbb{Z} / f)^{*}\right\}$ span the vector space

$K_{3} A \otimes \mathbb{Q}$. Any relation between them is a consequence of the relation 2) in Theorem 6.1.

Combining these results with the explicit formulas for $L^{\prime}(-1, \chi)$ given at the end of $\S 5$, one obtains a proof of conjecture 3.11 for all abelian representation $V$ of $\operatorname{Gal}(\overline{\mathbb{Q}} / \mathbb{Q})$ when $m=1$.

Proof of 6.1. To exhibit elements in $K_{3} A \otimes \mathbb{R}$ we will use Borel's isomorphism $(2.5): K_{3} A \otimes \mathbb{R} \underset{\lambda_{3}}{\stackrel{\sim}{\longrightarrow}}(Y \otimes \mathbb{R})^{-}$. The elements

$\left\{\phi^{-\operatorname{Frob}(a)}-\phi^{\operatorname{Frob}(a)}: a \in(\mathbb{Z} / f)^{*} / \pm 1\right\}$ give a basis for the space $(Y \otimes \mathbb{R})^{-}$. Associating to each basis element a copy of hyperbolic 3 -space

$$
H=\left\{(x+i y, z) \in \mathbb{C} \times \mathbb{R}_{+}^{*}\right\}
$$

we may specify an element $\alpha \in K_{3} A \otimes \mathbb{R}$ by giving a collection of 3 -simplices (or, more generally, 3 -chains) in $H$ :

$$
\Delta(\alpha)=\left(\ldots, \Delta_{a}(\alpha), \ldots\right)_{a \in(\mathbb{Z} / f)^{*}} / \pm 1
$$

Then $\lambda_{3}\left(\Delta_{a}(\alpha)\right)=\left(\ldots\right.$, vol $\left.\Delta_{a}(\alpha), \ldots\right)$, where the volume is computed with respect to the invariant 3 -form $(d x d y d z) / \pi z^{3}$. It is sufficient, for the purpose of representation, to use ideal simplices $\Delta_{a}(\alpha)$ with four vertices on the boundary.

What condition will insure that $\Delta(\alpha)$ lies in the rational vector space $K_{3} A \otimes$ $\mathbb{Q}$ ? We might hope this would hold when the four vertices of $\Delta(\alpha)$ lie in the rational boundary $\mathbb{P}^{1}(F) \hookrightarrow\left(\ldots, \mathbb{P}^{1}(\mathbb{C})_{a}, \ldots\right)$. At least this condition makes it easy to compute the Galois action: Since Borel's isomorphism commutes with $G=\operatorname{Gal}(F / \mathbb{Q})$, the Galois group acts on these cycles via its action on the vertices in $\mathbb{P}^{1}(F)$. 
However, the rational boundary assumption does not suffice to give a rational cycle: There is a further geometric invariant which must vanish. If we normalize the vertices of $\Delta(\alpha)$ to be at the points $\left\{\infty, 0,1, z_{\alpha}\right\}$ with $z_{\alpha} \in F-\{0,1\}$, Thurston has shown that $\alpha$ is a rational cycle iff $\left(z_{\alpha}\right) \wedge\left(1-z_{\alpha}\right)=0$ in the rational vector space $\Lambda^{2}\left(F^{*} \otimes \mathbb{Q}\right)$. More generally, the chain $\sum_{i} a_{i} \Delta\left(\alpha_{i}\right)$ with $a_{i} \in \mathbb{Q}$ gives a rational cycle iff

$$
\sum_{i} a_{i} \cdot\left(z_{\alpha_{i}}\right) \wedge\left(1-z_{\alpha_{i}}\right)=0 \quad \text { in } \quad \Lambda^{2}\left(F^{*} \otimes \mathbb{Q}\right)
$$

The condition is certainly met when $z_{\alpha}$ is a root of unity! Moreover, the volume of an ideal simplex with vertices $\left\{\infty, 0,1, e^{2 \pi i a / f}\right\}$ is $\frac{1}{\pi} \operatorname{dilog}\left(e^{2 \pi i a / f}\right)$. Taking $C_{f}(a)$ to be the element in $K_{3} A \otimes \mathbb{Q}$ represented by the ideal simplex with vertices $\left\{\infty, 0,1, \phi^{-1}\left(e^{2 \pi i a / f}\right)\right\}$ in $\mathbb{P}^{1}(F)$, we get the theorem.

Proof of 6.2. Since Borel's isomorphism $K_{3} A \otimes \mathbb{C} \underset{\lambda_{3}}{\stackrel{\sim}{\longrightarrow}}(Y \otimes \mathbb{C})^{-}$is $G$-equivariant the $\chi^{-1}$-eigenspace of $K_{3} A \otimes \mathbb{C}$ is zero when $\chi$ is even and one-dimensional when $\chi$ is odd. By 6.1 we see that the element $C_{\chi}$ always lies in the $\chi^{-1}$-eigenspace; it suffices to show $C_{\chi} \neq 0$ when $\chi$ is odd.

But the $\phi$-coordinate of $\lambda_{3}\left(C_{\chi}\right)$ is equal to

$$
\frac{1}{\pi} \sum_{(\mathbb{Z} / f)^{*}} \chi(a) \operatorname{dilog}\left(e^{2 \pi i a / f}\right)
$$

by 6.1 . This character sum is nonzero by 5.2 . Hence $C_{\chi} \neq 0$.

Now observe that the elements $C_{f}(a)$ with $(a, f)=1$ span $K_{3} A \otimes \mathbb{C}$, as they span all the nontrivial eigenspaces for $G$. Hence they span $K_{3} A \otimes \mathbb{Q}$; since this space has dimension $\phi(f) / 2$, there can be no additional relations beyond those in 6.1. This completes the Proof of 6.2 .

Finally, we will discuss the norms of elements in $K_{3} A \otimes \mathbb{Q}$ to abelian subfields $E$ of $F$. Let $B$ be the ring of integers in $E$ and $H=\operatorname{Gal}(F / E)$. The inclusion $B_{i} A$ induces a $G$-homomorphism $i_{*}: K_{3} B \rightarrow K_{3} A$. Using Borel's isomorphism, one can show

Lemma 6.3. The map $i_{*} \otimes 1: K_{3} B \otimes \mathbb{Q} \rightarrow K_{3} A \otimes \mathbb{Q}$ is injective and has image equal to the subspace $\left(K_{3} A \otimes \mathbb{Q}\right)^{H}$. 
Now for $\sigma \in G / H$ let $a_{\sigma}$ be any lifting of $\sigma$ to $G$. Define the element

$$
C_{f}(\sigma)=\sum_{a \in H} C_{f}\left(a a_{\sigma}\right)=\sum_{a \in H} C_{f}\left(a_{\sigma}\right)^{\operatorname{Frob}(a)} .
$$

By Lemma 6.3, this gives an element of $K_{3} B \otimes \mathbb{C}$ which is independent of the lifting chosen.

\section{Corollary 6.4.}

1) For $\tau \in G / H=\operatorname{Gal}(E / \mathbb{Q}): C_{f}(\sigma)^{\tau}=C_{f}(\sigma \tau)$,

2) $C_{f}(\bar{\sigma})=-C_{f}(\sigma)$,

3) The elements $\left\{C_{f}(\sigma): \sigma \in \operatorname{Gal}(E / \mathbb{Q})\right\}$ span the vector space $K_{3} B \otimes \mathbb{Q}$. All relations between them are a consequence of 2$)$.

This follows immediately from the definition of $C_{f}(\sigma)$ and Theorem 6.2. For a given abelian extension $E / \mathbb{Q}$, we may take $f$ to be the non-Archimedian part of the conductor of $E$.

\section{References}

1. Bloch, S.: Lectures on algebraic cycles. Duke Univ. Math. Series 4 (1980).

2. Borel, A.: Cohomology de $S L_{n}$ et valeurs de fonctions zeta. Annali Scuola Normale Supériore Pisa (1977).

3. Borel, A.: Stable real cohomology of arithmetic groups. Annales E.N.S. (1974).

4. Borel, A.: Sur la cohomologie des espaces fibrés principaux et des espaces homogènes de groups de Lie compacts. Annals of Math. (1953).

5. Borevitch, Z., Shafarevitch, I.: Number theory. Academic Press (1966).

6. Deligne, P.: Valeurs de fonctions $L$ et périodes d'intégrales. Proceedings of the American Mathematical Society, Summer Institute (1979).

7. Dupont, J.: Simplicial de Rham cohomology and characteristic classes of flat bundles. Topology (1976). 
8. Lichtenbaum, S.: Values of zeta-functions, étale cohomology, and algebraic $K$-theory. Springer Lecture Notes in Math. 342 (1973).

9. Quillen, D.: Cohomology of groups. International Congress of Math. Nice (1970).

10. Tate, J.: Les conjectures de Stark sur les Fonctions $L$ d'Artin en $s=0$. Birkhäuser Prog. in Math. 47 (1984).

\section{Additional Reference}

1. Beilinson, A.A. Higher regulators and values of $L$-functions. J. Soviet Math. 30 (1985), 2036-2070.

Leverett Professor of Mathematics Dean of Harvard College University Hall (617) 495-1560

Email:gross@math.harvard.edu 